\title{
Effects of Adding a Combined Infraorbital and Infratrochlear Nerve Block to General Anaesthesia in Septorhinoplasty
}

This article was published in the following Dove Press journal:

Journal of Pain Research

\section{Cem Kıvılcım Kaçar $\mathbb{D}$ \\ Osman Uzundere $\mathbb{D}$ \\ Fikret Salık (iD \\ Mesut Akgündüz (D) \\ Esra Aktiz Bıçak \\ Abdulkadir Yektaș (D)}

Anaesthesiology and Reanimation Clinic, Gazi Yașargil Education and Research

Hospital, TR Health Sciences University, Diyarbakır, Turkey
Correspondence: Abdulkadir Yektaș Anaesthesiology and Reanimation Clinic, Gazi Yașargil Education and Research Hospital, TR Health Sciences University, Diyarbakır, Turkey

$\mathrm{Tel} / \mathrm{Fax}+90-4 / 2-2580060$ ext 3/48

Email akyektas@hotmail.com
Purpose: In this study, we evaluated the hypothesis that preoperative bilateral infraoptic nerve (ION) and infratrochlear nerve (ITN) blocks under general anesthesia with sevoflurane and remifentanil reduced the incidence of emergence agitation (EA), pain scores, and the analgesic consumption after the septorhinoplasty.

Patients and Methods: Our study was conducted as a prospective randomized, double-sided blind study. Fifty-two patients whose septorhinoplasty operation was planned under general anesthesia were included in the study. Patients were randomly distributed to either the ION and ITN blocks were performed. Group 1: Bilateral ION and ITN blocks were performed; Group 2: ION and ITN blocks were not performed. Duration of the surgery and anesthesia, Riker Sedation-Agitation Scale (RSAS) score, EA presence, duration of postoperative analgesia, numerical rating scale (NRS) scores, and cumulative dexketoprofen consumption were recorded. Results: The RSAS score, NRS score and cumulative dexketoprofen consumption of the patients in Group 1 were statistically significantly lower than the patients in Group 2 $(\mathrm{p}<0.05)$. It was also found that patients in Group 1 (n: 8/26) had less EA compared to patients in Group 2 (n: 16/26) and this difference was statistically significant (p: 0.026). Postoperative analgesia duration of patients in Group 1 was found to be statistically significantly higher than patients in Group 2 (p: <0.001). In addition, the number of patients given postoperative dexketoprofen in Group 1 (n: 8/26) was found to be statistically significantly lower than patients in Group 2 (n: 25/26). (p: <0.001).

Conclusion: Bilateral ION and ITN blocks in septorhinoplasty operation is an effective, reliable and simple technique in the treatment of postoperative pain.

Keywords: emergence agitation, infraorbital nerve block, infratrochlear nerve block, postoperative pain, septorhinoplasty

\section{Introduction}

Pain management is a crucial component of postoperative care. ${ }^{1}$ Pain cannot be managed well in approximately $80 \%$ of postoperative patients. ${ }^{2}$ Inadequate postoperative pain management may increase thromboembolic and pulmonary complications, length of hospital stay, opioid use, recovery time, development of chronic pain, mortality, and morbidity. ${ }^{1-3}$ More so, inadequate pain management directly affects the success of the surgical procedure and creates an uncomfortable morbid condition during the postoperative period. ${ }^{3,4}$

Septorhinoplasty is one of the most frequently used nasal surgical procedures. ${ }^{3}$ Severe pain may occur in the postoperative period of septorhinoplasty due to soft 
tissue injury and osteotomies. ${ }^{5}$ On a numerical rating scale $(\mathrm{NRS})^{6}$ from 0 to 10 , a pain score of $>3$ in $45 \%$ of patients and a pain score of $>6$ in almost $30 \%$ of patients were reported after a septorhinoplasty operation. ${ }^{7}$ In their study examining the postoperative pain of patients undergoing rhinoplasty operation, Demir et al accepted patients with an NRS score of $\geq 5$ as patients with postoperative pain and applied analgesics to them. ${ }^{8}$ The latest practice guidelines published by the American Society of Anesthesiologists (ASA) for perioperative acute pain management recommend the use of multimodal analgesia and regional blockages where possible. ${ }^{9}$ The use of opioid analgesics in the recovery room is very effective for pain management in these patients but has negative effects such as sedation, respiratory depression, nausea and vomiting. ${ }^{10,11}$ Also, the side effects of opioid analgesics may delay the patient's discharge. ${ }^{11}$ Peripheral nerve blocks are increasingly used for postoperative analgesia. Peripheral nerve blocks offer advantages of less tissue edema, a wider area of anesthesia and lower pain in the surgical site. ${ }^{12}$

The infraorbital nerve (ION) is a branch of the maxillary part of the trigeminal nerve. This nerve innervates the skin of the nose and septum mobile nasi. The infratrochlear nerve (ITN) innervates the root of the nose and is an extraconal branch of the nasociliary nerve, which is a branch of the ophthalmic portion of the trigeminal nerve. ${ }^{13}$ Together with general anesthesia, these two peripheral nerve blocks have been reported to facilitate pain management, reduce complications, and reduce anesthetic agent consumption after nasal procedures. ${ }^{7,12,14}$

Emergence agitation (EA) is a clinical condition characterized by agitation, confusion, disorientation and aggressive behavior in the early stage of recovery from general anesthesia. ${ }^{15}$ Although the etiology of EA is not well understood, known risk factors are age (young), sex (male), smoking history, benzodiazepine premedication, sevoflurane anesthesia, postoperative pain, tracheal tube and/or urinary catheter. ${ }^{13}$ The incidence of EA can reach up to $55.4 \%$ in adults after ear, nose and throat surgery. ${ }^{15}$ EA can lead to serious consequences such as various injuries, self-extubation, hemorrhage, increased pain, removal of catheters, respiratory depression, nausea/ vomiting, increased blood pressure/heart rate (HR), and myocardial oxygen consumption. ${ }^{16}$ Pain management can be an effective preventive measure for EA. ${ }^{17}$

In this study, we evaluated the hypothesis that preoperative bilateral ION and ITN blocks under general anesthesia with sevoflurane and remifentanil reduced the incidence of EA, pain scores, and the analgesic consumption after the septorhinoplasty.

\section{Patients and Methods Patients}

Our study was conducted as a prospective randomized, double-sided blind study between February 2020 and April 2020 in the operating room of the Diyarbakır Gazi Yaşargil Training and Research Hospital at the University of Health Sciences. We started the study after getting approval (422/2020) from the ethics committee of our hospital. Our study was conducted by the 2008 Helsinki Declaration and written informed consent was obtained from all patients. Fifty-two patients between the ages 18-65 who had ASA physical status ${ }^{18}$ I-II and whose septorhinoplasty operation was planned under general anesthesia were included in the study. Pregnancy, cancer, known local anesthetic drug allergy, preoperative chronic pain, inability to provide informed consent, body mass index (BMI) $35>\mathrm{kg} / \mathrm{m} 2$ and coagulopathy were considered as exclusion criteria. Patients were randomly distributed to either the ION and ITN blocks were performed. Group 1: ION and ITN blocks were performed; Group 2: ION and ITN blocks were not performed. The randomization sequence was generated by a computerized random number generator and sealed in numbered envelopes. The anesthesiologist who performed nerve blocks and the anesthesiologist who performed extubation and postoperative care were different.

\section{General Anesthesia Maintenance}

After the patients were taken to the operating room, venous cannulation was performed on the back of the hand with an $18 \mathrm{G}$ intravenous (IV) cannula. $10 \mathrm{~mL} / \mathrm{kg} / \mathrm{h}$ IV isotonic $\mathrm{NaCl} 0.9 \%$ infusion was started. $5 \mathrm{mg}$ IV dexamethasone (Dekort, Deva, Istanbul, Turkey) was administered to prevent postoperative nausea and vomiting (PONV) and surgical edema and ecchymosis. The patients were continuously monitored with electrocardiography, pulse oximeter and noninvasive blood pressure measurements recorded every 5 minutes. Preoxygenation was done with a facemask for three minutes. $2-2.5 \mathrm{mg} / \mathrm{kg}$ IV propofol (Propofol 1\% Fresenius ${ }^{\circledR}$; Fresenius Kabi Medicine, Istanbul, Turkey), $0.6 \mathrm{mg} / \mathrm{kg}$ IV rocuronium (Esmeron ${ }^{\circledR}$, Merck Sharpoo Dohme (MSD) Pharmaceuticals Ltd., Germany), and $1 \mu \mathrm{g} / \mathrm{kg}$ IV fentanyl (Talinat, Vem, Istanbul, Turkey) was administered in anesthesia 
induction. For all patients in our study, we used Dräger Primus (Dräger AG, Lübeck, Germany) as the anesthesia device and monitor and Sevoflurane vaporizer (Drager Vapor 2000, Lubeck, Germany) as a vaporizer. After intubation with the appropriately sized cuffed endotracheal tube (Kaishou Inc., China), mechanical ventilation was initiated with a mixture of $50 \%$ air and $50 \%$ oxygen, and the minute ventilation was adjusted to keep the end-tidal carbon dioxide between 30 and $35 \mathrm{mmHg}$. The maintenance of anesthesia was achieved by $0.05-0.2 \mu \mathrm{g} / \mathrm{kg} / \mathrm{min}$ IV infusion of remifentanil (Rentanil ${ }^{\circledR}$, VEM, Ankara, Turkey) and 1.5-2 end-tidal minimal alveolar concentration value of sevoflurane (Sevorane liquid 100\%, Abbott Laboratory, Istanbul, Turkey). For antimicrobial prophylaxis, 2 gr IV cefazolin (Cefazol, Mustafa NevzatIlac, Istanbul, Turkey) was administered. Necessary interventions for intraoperative hemodynamic changes were performed by the anesthesiologist who followed the patient.

\section{Bilateral Infraorbital and Infratrochlear Nerve Blocks}

After induction, bilateral ION and ITN blocks were applied to the patients in Group 1 by a single experienced anesthesiologist. ${ }^{7}$ In the supine position, the patient's head was placed on a central line. The infraorbital ridge was palpated. Thus, the ION block was performed with the extraoral approach by determining the location of the infraorbital foramen. A $25 \mathrm{G}$ needle (Ayset Inc, Turkey) was inserted laterally into the nostril, then moved forward $1-2 \mathrm{~cm}$ until it was felt under the finger localizing the location of the foramen to prevent globe injuries. Taking care not to inject into the foramen itself, $4 \mathrm{~mL}$ of $0.5 \%$ bupivacaine (Buvasin 0.5\%, $20 \mathrm{~mL}$, VemIlac, Istanbul, Turkey) was slowly injected after negative blood aspiration was confirmed. The ITN block was performed by placing the needle $1 \mathrm{~cm}$ above the inner canthus and targeting the junction of the orbit and nasal bone. After the negative blood aspiration, $1 \mathrm{~mL}$ of $0.5 \%$ bupivacaine was injected. The same amounts of bupivacaine were injected into the opposite side using the same techniques. One-minute pressure was applied to the injection points to prevent hematomas. ${ }^{7,13}$

\section{Operation and Extubation}

The surgical site including the nasal septum, nasal concha, columella, and nasal dorsum was then infiltrated with $10 \mathrm{~mL}$ of $1 \%$ lidocaine and $10 \mu \mathrm{g} / \mathrm{mL}$ of epinephrine for local anesthesia and decrease intraoperative bleeding. Septorhinoplasty was performed by open approach. Bone and cartilaginous septum were carved to improve nasal airflow. If necessary, an extracorporeal technique was used. Dorsal hump was removed, and then lateral osteotomies were performed on both sides of the nose. At the end of the operation, the nasal tampon was performed using silicone internal nasal splint (Unosplint; Genco Medical Devices, Izmir, Turkey). Thirty minutes before the end of the operation, all patients were administered 1g IV paracetamol (Parol, Atabay, Istanbul, Turkey) for postoperative pain management and $4 \mathrm{mg}$ IV ondansetron (Zofer, Adeka; Istanbul, Turkey) for PONV prophylaxis. At the end of the operation, oral aspiration was performed, and all patients were administered $0.01 \mathrm{mg} / \mathrm{kg}$ IV atropine and $0.06 \mathrm{mg} / \mathrm{kg}$ IV neostigmine (Neostigmine; Adeka Inc., Istanbul, Turkey) for reversing the neuromuscular block. Tracheal extubation was performed after the patients responded to spontaneous ventilation and verbal commands. During the period from extubation to the discharge from postanesthesia care unit (PACU), patients' agitation status was evaluated using the Riker SedationAgitation Scale (RSAS). ${ }^{19}$ The Riker Sedation-Agitation Scale uses a numeric score from 1 to 7 to assess the level of patient sedation and is especially adapted to warn the clinician of "unarousable" and "dangerous agitation" levels of patient sedation. ${ }^{19}$ The highest RSAS score detected during this period was recorded. An RSAS score of $\geq 5$ was defined as EA. ${ }^{13}$

\section{Postoperative Period}

The patients were then sent to PACU. Patients were monitored in PACU. Aldrete score is the most widely used tool to clinically assess the physical status of patients recovering from anesthesia. ${ }^{20}$ The patients with an Aldrete score of $>9$ and no significant postanesthetic complications were taken into the ward. Physician who evaluated the postoperative pain had no knowledge of the patient groups. Postoperative pain was evaluated using a NRS ranging from 0 (no pain) to 10 (maximum imaginable pain). ${ }^{6}$ The highest NRS scores of the patients were recorded in 0-2 hours, 2-8 hours and 8-24 hours after reaching PACU (0 hours). In the case of postoperative pain with an NRS score of $\geq 5,50 \mathrm{mg}$ IV dexketoprofen (Deksalgin; Nobel Ilac, Istanbul, Turkey) was administered. The time until the first postoperative dexketoprofen was administered was accepted 
as the duration of postoperative analgesia. In the case of PONV, $4 \mathrm{mg}$ IV ondansetron was administered.

\section{Recorded Data}

The recorded demographic and physiological data of the patients were age, gender, BMI, ASA, smoking, mean arterial pressure (MAP) and HR (before induction, before skin incision, during surgery and before extubation). Also, duration of the surgery and anesthesia, RSAS score, EA presence, duration of postoperative analgesia, NRS score (in 0-2 hours, 2-8 hours, and 8-24 hours of postoperative period), and cumulative dexketoprofen consumption (in 0-2 hours, 2-8 hours, and 8-24 hours of postoperative period) were recorded.

\section{Statistical Analysis}

Statistical data were expressed as mean and standard deviation, while categorical data were expressed as frequency and percentage. Comparison of categorical data in the groups was done with chi-square and Fisher's Exact Test, the results were given as \%n. Skewness and Kurtosis tests were used to determine if the numerical data fit the normal distribution. While the data fitting the normal distribution were evaluated with the Student's $t$-test, MannWhitney U and Kruskal Wallis tests were used to compare the data differ from the normal distribution. G-Power version 3.1.9.4 (Universität Kiel, Germany) program was used to calculate the sample size. The two-tailed alpha error was taken as 0.05 , power as 0.80 and effect size as 0.8 , and based on a previous study ${ }^{7}$ the allocation ratio was accepted as $\mathrm{N} 2 / \mathrm{N} 1: 1$. The minimum number of patients to be included in the study was calculated as 52 . SPSS 16.0 for Windows (SPSS Inc., Chicago, IL, USA) was used for other statistical analyses. $\mathrm{p}<0.05$ was considered statistically significant.

\section{Results}

Fifty-two patients were included in the study. The patients were divided into two groups and compared in terms of demographic, clinical and intraoperative characteristics. The duration of surgery and anesthesia of the patients in Group 1 (64.23 $\pm 9.66 \mathrm{~min} . / 76.88 \pm 11.43 \mathrm{~min}$.) compared to the patients in Group $2(56.92 \pm 9.06 \mathrm{~min} . / 67.69 \pm 9.61$ $\mathrm{min}$.) was found to be longer and this difference was found to be statistically significant ( $\mathrm{p}$ values, respectively: 0.007 ; 0.003) (Table 1). There was no statistically significant difference between the groups in terms of MAP and HR before induction, before skin incision, during surgery, and before extubation and also no significant difference in terms of age, gender, BMI, ASA and smoking (Table 1; Figure 1).

It was found that the RSAS score, NRS score and cumulative dexketoprofen consumption of the patients in Group 1 were statistically significantly lower than the patients in Group $2(\mathrm{p}<0.05)$ (Figures 2-4).

It was found that patients in Group 1 (n: 8/26) had less EA compared to patients in Group 2 (n: 16/26) and this difference was statistically significant (p: 0.026). Postoperative analgesia duration of patients in Group 1 was found to be statistically significantly higher than patients in Group 2 (p: <0.001). In addition, the number of patients given postoperative dexketoprofen in Group 1 (n: 8/26) was found to be statistically significantly lower than patients in Group 2 (n: 25/26). (p: <0.001) (Table 2). We did not experience any complications due to the nerve blocks we applied.

\section{Discussion}

The results of this study show that postoperative pain management through bilateral ION and ITN blocks decreases RSAS score, EA incidence, postoperative analgesic consumption, and postoperative NRS scores in patients undergoing a septorhinoplasty operation under general anesthesia of sevoflurane/remifentanil. Intraoperative hemodynamic parameters were similar in both groups.

Choi et al performed bilateral ION and ITN blocks 30 minutes before the operation for postoperative analgesia in patients who were to undergo a septorhinoplasty operation. ${ }^{13}$ Wang et al examined the postoperative analgesic efficacy of the ION block in the cleft lip operation and applied bupivacaine in the block group and saline in the control group after induction. ${ }^{21}$ In both studies, there was not any difference between the block group and the control groups in terms of the duration of surgery and anesthesia. ${ }^{13,21}$ In the present study, the duration of surgery and anesthesia in patients in the block group was statistically longer than in the control group ( $p$ values, respectively: $0.007 ; 0.003$ ). We think that this difference in the duration of anaesthesia occurred because we applied the nerve blocks after the induction and did not perform any placebo injection to the control group. However, the duration of surgery in Group 1 was unexpectedly longer than in Group 2. We think this is purely coincidental. 
Table I Comparison of Groups in Terms of Demographic and Intraoperative Features

\begin{tabular}{|c|c|c|c|}
\hline & Group I $(n=26)$ & Group $2(n=26)$ & \multirow[t]{2}{*}{$\mathbf{p}$} \\
\hline & Mean $\pm S D *$ & Mean $\pm S D *$ & \\
\hline Age (year) & $27.38 \pm 7.09$ & $24.5 \pm 5.5$ & 0.13 \\
\hline Body mass index $(\mathrm{kg} / \mathrm{m} 2)$ & $24.75 \pm 3.71$ & $23.24 \pm 3.49$ & 0.111 \\
\hline Duration of surgery (min) & $64.23 \pm 9.66$ & $56.92 \pm 9.06$ & $0.007^{* *}$ \\
\hline \multirow[t]{2}{*}{ Duration of anesthesia (min) } & $76.88 \pm 11.43$ & $67.69 \pm 9.61$ & $0.003 * *$ \\
\hline & n(\%) & n(\%) & \\
\hline \multicolumn{4}{|l|}{ Gender } \\
\hline Female & $15(57.7)$ & $13(50)$ & 0.57 \\
\hline Male & II (42.3) & $13(50)$ & \\
\hline \multicolumn{4}{|l|}{ ASA $* * *$ Class } \\
\hline 1 & $16(6 \mid .5)$ & $17(65.4)$ & 0.77 \\
\hline II & $10(38.5)$ & $9(34.6)$ & \\
\hline \multicolumn{4}{|l|}{ History of smoking } \\
\hline+ & $9(34.6)$ & $9(34.6)$ & 1.0 \\
\hline- & $17(65.4)$ & $17(65.4)$ & \\
\hline
\end{tabular}

Notes: *Mean \pm standard deviation; **Statistically significant; ***American Society of Anesthesiologists.

The incidence of EA in adults has been reported to be $21.3 \% .^{22}$ The exact etiology of EA is uncertain. No factor can explain the cause of EA, but the pain is considered an important contributing factor in EA. A positive correlation between EA and pain has been reported in the literature. ${ }^{21}$ Septorhinoplasty operation is associated with high postoperative pain scores. ${ }^{10,23}$ Choi et al reported a strong correlation between pain and EA in septorhinoplasty patients. ${ }^{13}$ It has been reported in the literature that EA may occur after general anesthesia with sevoflurane in the absence of painful stimuli. This suggests that the intrinsic effect of sevoflurane is associated with EA. ${ }^{21}$ It has been suggested that rapid elimination of sevoflurane due to its low solubility in the blood can cause EA. ${ }^{24}$ Incidence rates of $55.4 \%$ and $54.3 \%$ for EA among nasal surgery patients have been reported in the literature. ${ }^{8,15}$ In our study, all patients received sevoflurane for general anesthesia and underwent septorhinoplasty which caused high postoperative pain and shortness of breath in the early postoperative period. Therefore, a high incidence of EA was expected in our patients. As expected, we found the incidence of EA to be $61.5 \%$ in the control group. We think that this result is compatible with the literature. ${ }^{8,15}$ Mild trauma in the early postoperative period may also adversely affect the operative results, especially since the nasal bones are broken during osteotomy in rhinoplasty. In this regard, it is important to reduce the risk of trauma by preventing EA in such patients. ${ }^{8}$ Adequate analgesia with regional blocks, opioids, and nonsteroidal anti-inflammatory drugs can prevent and treat agitation that occurs even in painless procedures. ${ }^{25}$ However, pharmacological prevention can cause residual sedation and hemodynamic changes, such as hypotension, hypertension, and bradycardia, resulting in long-term PACU stay and high cost of hospital stay. ${ }^{13}$ In the present study, while EA was $61.5 \%$ in the control group, it was decreased to $30.8 \%$ by the pain management with a peripheral nerve block.

Sensory nerves of the face in the field of septorhinoplasty surgery include ION and ITN. ${ }^{26,27}$ Bilateral ION block has been reported to reduce postoperative pain scores. $^{12,14}$ In a study of 40 patients who underwent nasal surgery, Mariano et al performed a bilateral ION block using an intraoral approach with $0.5 \%$ bupivacaine following anesthesia induction, resulting in a small but significant reduction in pain scores. ${ }^{14}$ Çekiç et al reported that the ION block performed with the combination of $0.25 \%$ levobupivacaine and $50 \mathrm{mg}$ tramadol provides 


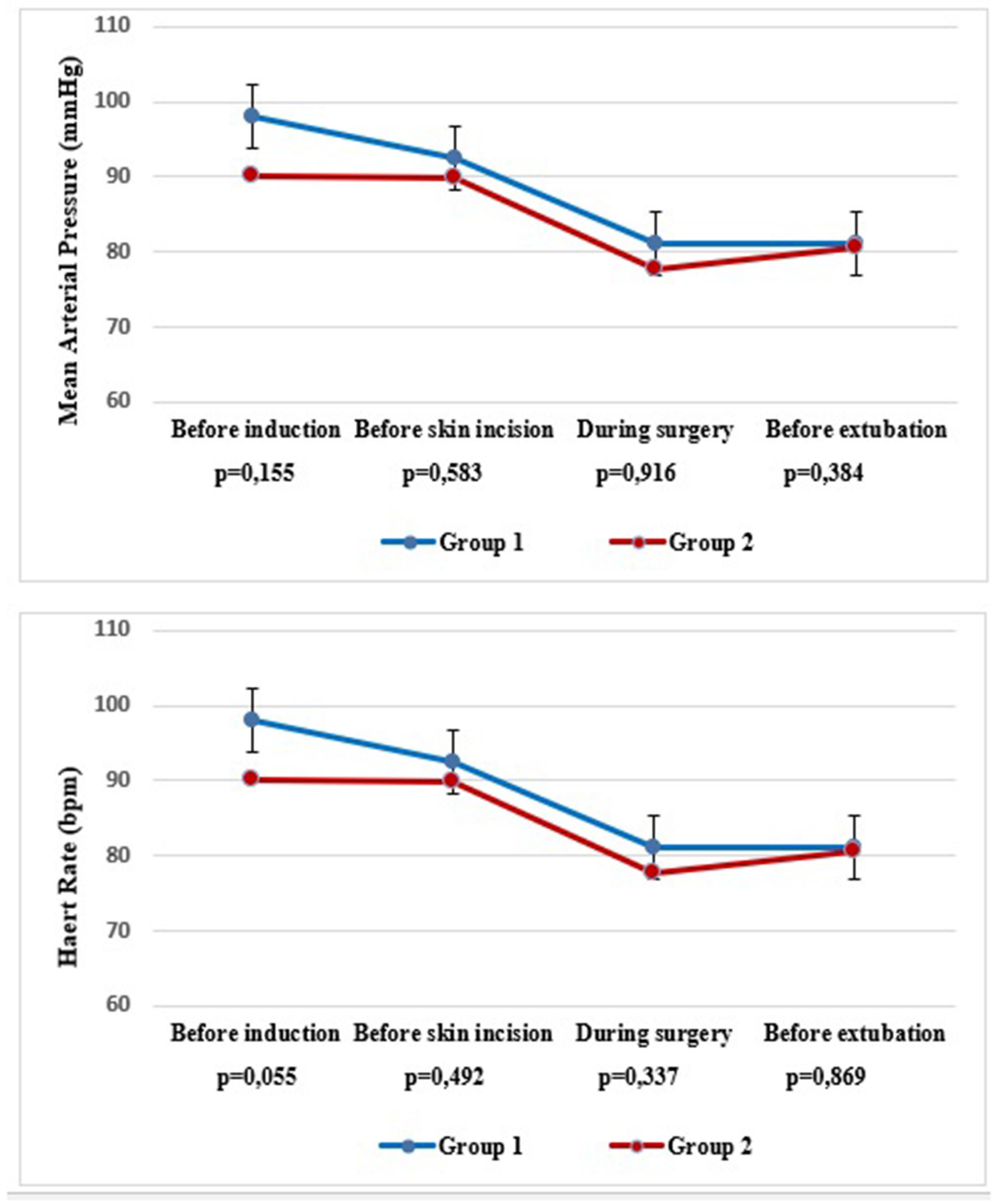

Figure I Comparison of the groups in terms of mean arterial pressure and heart rate.

effective postoperative analgesia in patients undergoing nasal surgery without any side effects. ${ }^{12}$ We found in the present study that the NRS score of patients in the block group (in $0-2$ hours, $2-8$ hours, and $8-24$ hours of postoperative period) was statistically significantly lower than the patients in the control group.

In a rhinoplasty study, Demir et al accepted patients with an NRS score of $\geq 5$ as patients with postoperative pain and applied analgesics to them. ${ }^{8}$ In our study, $1 \mathrm{~g} \mathrm{IV}$ paracetamol was administered to both groups 30 minutes before extubation, and $50 \mathrm{mg}$ IV dexketoprofen in the case of postoperative pain with an NRS score of $\geq 5$. In the present study, the time until the first postoperative dexketoprofen was given was accepted as the duration of postoperative analgesia. Postoperative analgesia duration of the patients in the block group was $19.58 \mathrm{~h}$ while $5.03 \mathrm{~h}$ for the patients in the control group (p: <0.001). We did not find any data on the duration of postoperative analgesia among similar studies in the literature, where ION and ITN blocks were performed for postoperative analgesia.

In two studies in which bilateral ION and ITN blocks were applied for postoperative analgesia in septorhinoplasty operation, it was reported that postoperative analgesic consumption decreased compared to the control 


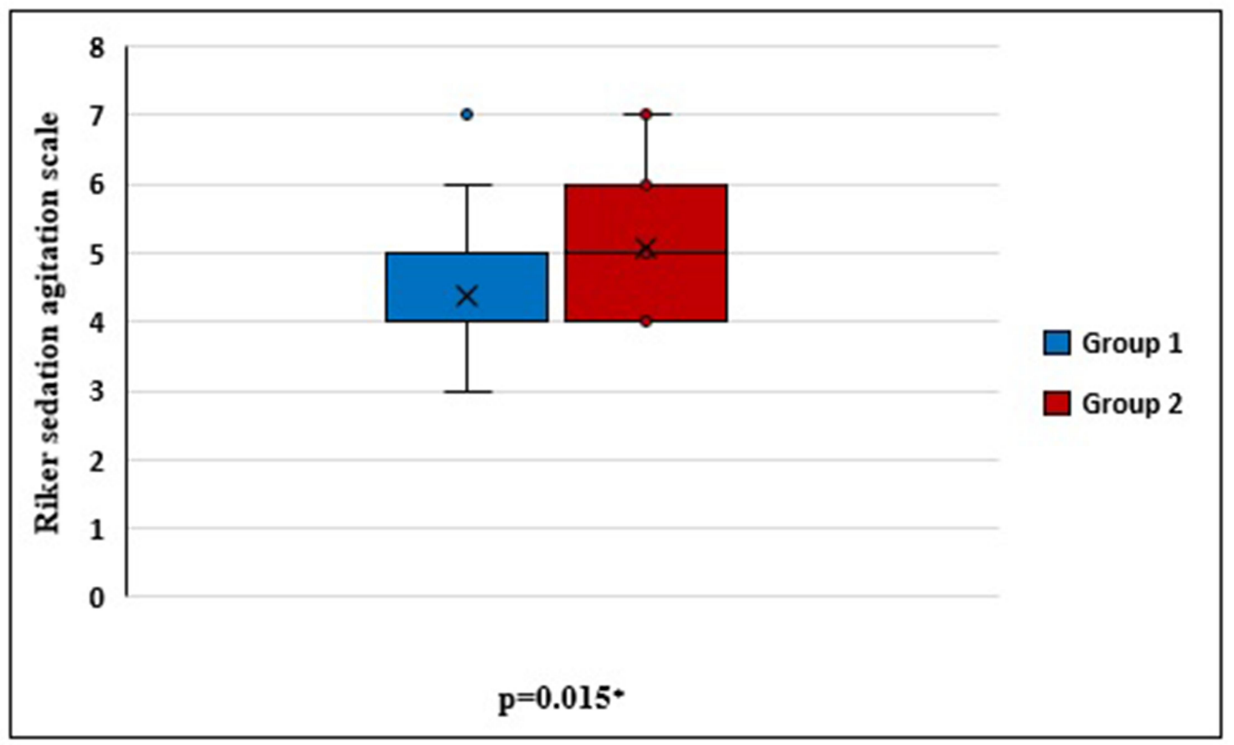

Figure 2 Comparison of groups in terms of Riker sedation-agitation scale. *Statistically significant.

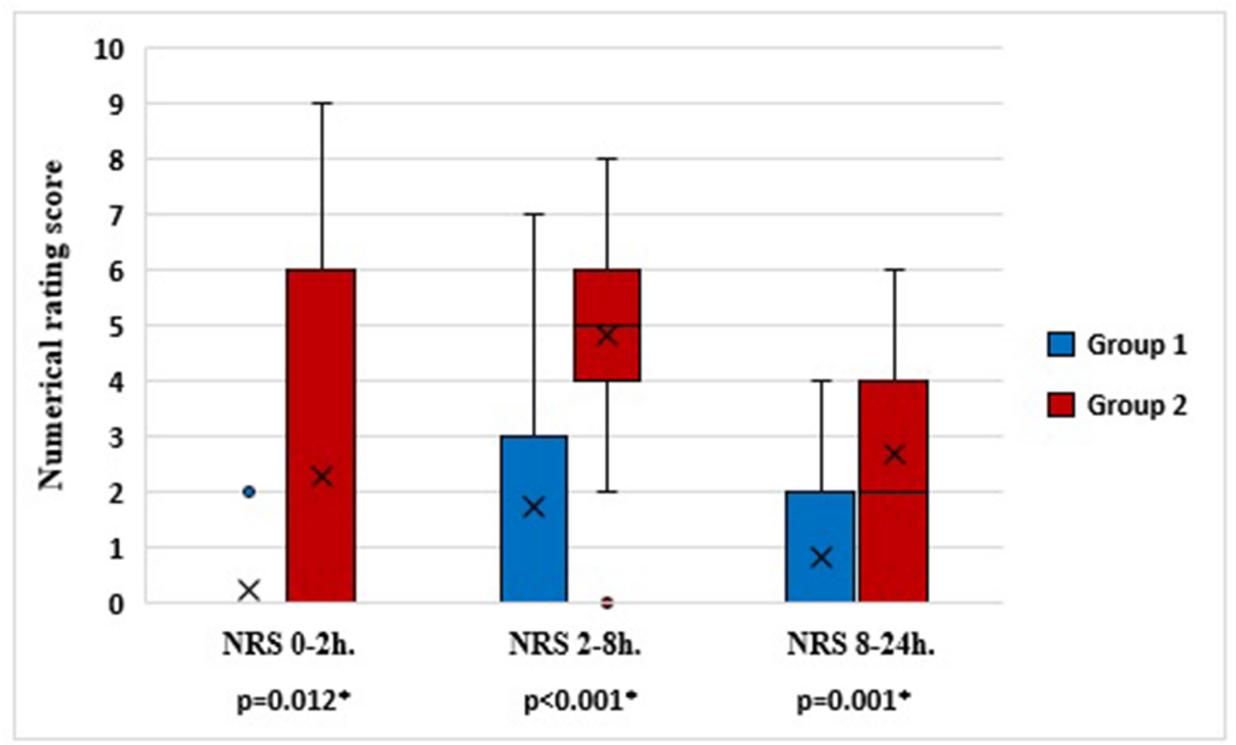

Figure 3 Comparison of groups in terms of postoperative numerical rating scale. *Statistically significant.

group. ${ }^{7,13}$ Çekiç et al reported a decrease in postoperative analgesic consumption in 2 groups in which they performed a bilateral ION block compared to the control group in nasal surgery. ${ }^{12}$ In line with these studies, ${ }^{7,12,13}$ we found that the cumulative dexketoprofen consumption (in 0-2 hours, 2-8 hours, and 8-24 hours of postoperative period) and the number of patients receiving postoperative dexketoprofen were statistically significantly lower in the block group than in the control group.
ION and ITN blocks in our study have some disadvantages. First, since nerve blocks were administered intraoperatively under general anesthesia, it was not possible to control them with preoperative sensory tests. Therefore, unsuccessful blocks may not have been detected. Secondly, the two nerve blocks performed do not cover all surgical sites. A more proximal level of nerve blocks, such as the maxillary nerve block and the nasociliary nerve block, has not been used due to difficulty in application and the need for neurostimulation. The limitation of 


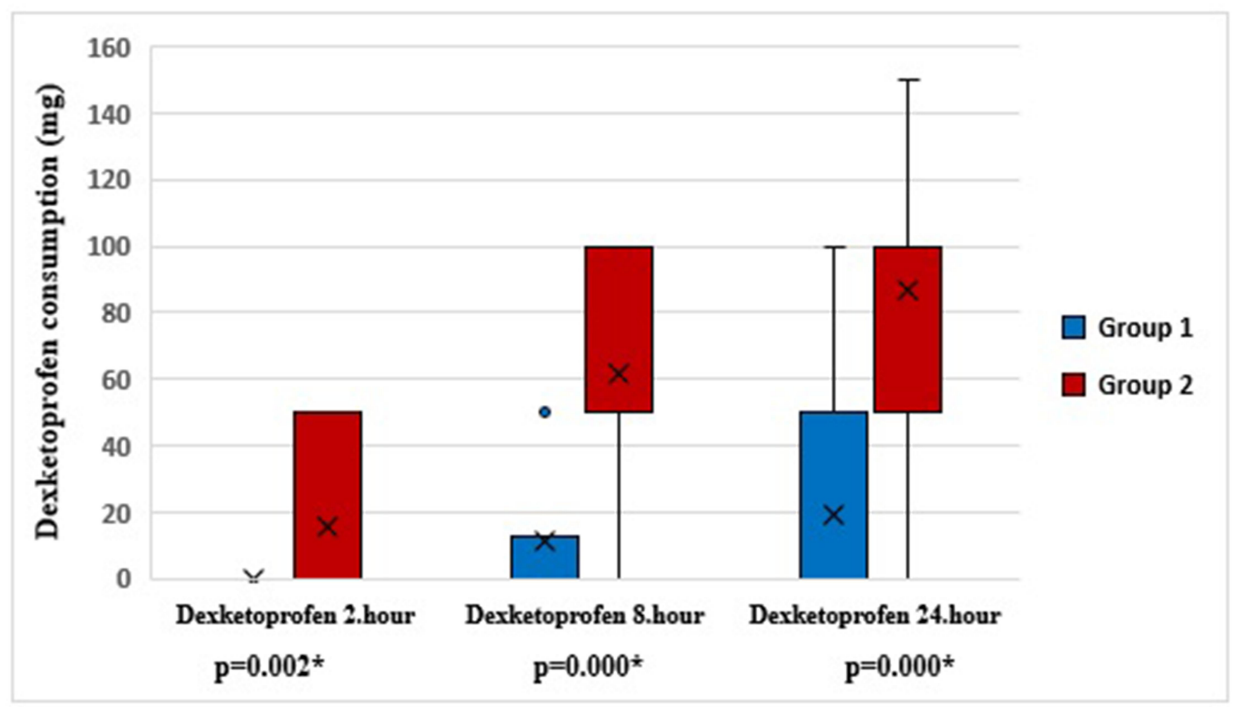

Figure 4 Comparison of groups in terms of postoperative dexketoprofen consumption. *Statistically significant.

our study was that it was a single-center study with a small sample size (52 patients). Further studies conducted as multicenter studies will give more confidence in the results.

\section{Conclusion}

In conclusion, our findings show that bilateral ION and ITN blocks during general anesthesia with remifentanil and sevoflurane, significantly decreased RSAS score, EA incidence, postoperative analgesic consumption, and the first $24 \mathrm{~h}$ postoperative NRS scores. This application also increased the duration of postoperative analgesia. Bilateral ION and ITN blocks in septorhinoplasty operation are an effective, reliable and simple technique in the treatment of postoperative pain.

\section{Abbreviations}

ASA, American Society of Anesthesiologists; BMI, body mass index; EA, emergence agitation; HR, heart rate; ION, infraoptic nerve; ITN, infratrochlear nerve; IV, intravenous; MAP, mean arterial pressure; NRS, numerical rating scale; PACU, postanesthesia care unit; PONV, postoperative nausea and vomiting; RSAS, Riker Sedation-Agitation Scale.

\section{Disclosure}

The authors report no funding and no conflicts of interest for this work.

Table 2 Comparison of the Groups in Terms of Duration of Postoperative Analgesia, Emergency Agitation and Number of Patients Receive Dexketoprofen

\begin{tabular}{|c|c|c|c|}
\hline & Group I $(n=26)$ & Group $2(n=26)$ & \multirow[t]{2}{*}{$\mathbf{p}$} \\
\hline & Mean $\pm S D *$ & Mean $\pm S D *$ & \\
\hline \multirow[t]{2}{*}{ Duration of postoperative analgesia (hour) } & $19.58 \pm 7.77$ & $5.03 \pm 4.50$ & $<0.00 I^{* *}$ \\
\hline & n (\%) & n (\%) & $\mathbf{p}$ \\
\hline \multicolumn{4}{|l|}{ Emergency agitation } \\
\hline+ & $8(30.8)$ & $16(61.5)$ & $0.026 * *$ \\
\hline- & $18(69.2)$ & $10(38.5)$ & \\
\hline \multicolumn{4}{|l|}{ Number of patients receive dexketoprofen } \\
\hline+ & $8(30.8)$ & $25(96.2)$ & $<0.00 I^{* *}$ \\
\hline - & I8 (69.2) & I (3.8) & \\
\hline
\end{tabular}

Notes: *Mean \pm standard deviation; **Statistically significant. 


\section{References}

1. Pourfakhr P, Khajavi MR, Jalali A, et al. Low-dose preoperative pregabalin improves postoperative pain management in septorhinoplasty surgery: a double-blind randomized clinical trial. Eur Arch Otorhinolaryngol. 2019;276(8):2243-2249. doi:10.1007/s00405-019-05448-y

2. Institute of Medicine (US) Committee on Advancing Pain Research, Care, and Education. Relieving Pain in America: A Blueprint for Transforming Prevention, Care, Education, and Research. Washington (DC): National Academies Press (US); 2011.

3. Çelik EC, Kara D, Koc E, Yayik AM. The comparison of single-dose preemptive intravenous ibuprofen and paracetamol on postoperative pain scores and opioid consumption after open septorhinoplasty: a randomized controlled study. Eur Arch Otorhinolaryngol. 2018;275 (9):2259-2263. doi:10.1007/s00405-018-5065-6

4. Ates I, Aydin ME, Ahiskalioglu A, Ahiskalioglu EO, Kaya Z, Gozeler MS. Postoperative analgesic efficacy of perioperative intravenous lidocaine infusion in patients undergoing septorhinoplasty: a prospective, randomized, double-blind study. Eur Arch Otorhinolaryngol. 2020;277(4):1095-1100. doi:10.1007/s00405020-05801-6

5. Kim DH, Kang H, Jin HJ, et al. Effect of piezoelectric osteotomy on postoperative oedema and ecchymosis after rhinoplasty. Clin Otolaryngol. 2019;44(6):968-974.

6. Jin J, Min S, Peng L, et al. No differences in the prevalence and intensity of chronic postsurgical pain between laparoscopic hysterectomy and abdominal hysterectomy: a prospective study. J Pain Res. 2020;13:1-9. doi:10.2147/JPR.S225230

7. Boselli E, Bouvet L, Augris-Mathieu C, et al. Infraorbital and infratrochlear nerve blocks combined with general anaesthesia for outpatient rhinoseptoplasty: a prospective randomised, double-blind, placebo-controlled study. Anaesth Crit Care Pain Med. 2016;35 (1):31-36. doi:10.1016/j.accpm.2015.09.002

8. Demir CY, Yuzkat N. Prevention of emergence agitation with ketamine in rhinoplasty. Aesthetic Plast Surg. 2018;42(3):847-853. doi:10.1007/s00266-018-1103-4

9. American Society of Anesthesiologists. Practice guidelines for acute pain management in the perioperative setting: an updated report by the american society of anesthesiologists task force on acute pain management. Anesthesiology. 2012;116(2):248-273. doi:10.1097/ ALN.0b013e31823c1030

10. Wittekindt D, Wittekindt C, Schneider G, et al. Postoperative pain assessment after septorhinoplasty. Eur Arch Otorhinolaryngol. 2012;269(6):1613-1621. doi:10.1007/s00405-011-1854-x

11. Lam DMH, Choi S-W, Wong SSC, et al. Efficacy of pregabalin in acute postoperative pain under different surgical categories a metaanalysis. Medicine (Baltimore). 2015;94(46):e1944. doi:10.1097/ MD.0000000000001944

12. Cekic B, Geze S, Erturk E, et al. A comparison of levobupivacaine and levobupivacaine-tramadol combination in bilateral infraorbital nerve block for postoperative analgesia after nasal surgery. Ann Plast Surg. 2013;70(2):131-134.

13. Choi H, Jung SH, Hong JM, et al. Effects of bilateral infraorbital and infratrochlear nerve block on emergence agitation after septorhinoplasty: a randomized controlled trial. J Clin Med. 2019;8(6):769. doi:10.3390/jcm8060769

Journal of Pain Research

\section{Publish your work in this journal}

The Journal of Pain Research is an international, peer reviewed, open access, online journal that welcomes laboratory and clinical findings in the fields of pain research and the prevention and management of pain. Original research, reviews, symposium reports, hypothesis formation and commentaries are all considered for publication. The manuscript

Submit your manuscript here: https://www.dovepress.com/journal-of-pain-research-journal
14. Mariano ER, Watson D, Loland VJ, et al. Bilateral infraorbital nerve blocks decrease postoperative pain but do not reduce time to discharge following outpatient nasal surgery. Can J Anaesth. 2009;56 (8):584-589. doi:10.1007/s12630-009-9119-5

15. Kim HJ, Kim DK, Kim HY, et al. Risk factors of emergence agitation in adults undergoing general anesthesia for nasal surgery. Clin Exp Otorhinolaryngol. 2015;8(1):46-51. doi:10.3342/ ceo.2015.8.1.46

16. Talih G, Yüksek A, Şahin E. Evaluation of emergence agitation after general anaesthesia in rhinoplasty patients: inhalation anaesthesia versus total intravenous anaesthesia. Am J Otolaryngol. 2020;41 (3):102387. doi:10.1016/j.amjoto.2020.102387

17. Dahmani S, Stany I, Brasher C, et al. Pharmacological prevention of sevoflurane- and desflurane-related emergence agitation in children: a meta-analysis of published studies. Br J Anaesth. 2010;104(2):216223. doi:10.1093/bja/aep376

18. Mayhew D, Mendonca V, Murthy BVS. A review of ASA physical status - historical perspectives and modern developments. Anaesthesia. 2019;74(3):373-379. doi:10.1111/anae.14569

19. Ault ML, Gould R. Pain control in the critically ill patient. In: Benzon HT, Molly RE, Fishman SM, Raja SN, Liu SS, editors. Essentials of Pain Medicine and Regional Anaesthesia (Second Edition). Churchill Livingstone; 2005:269-276.

20. Phillips NM, Street M, Kent B, et al. Post-anaesthetic discharge scoring criteria: key findings from a systematic review. Int $J$ Evid Based Healthc. 2013;11(4):275-284. doi:10.1111/17441609.12044

21. Wang H, Liu G, Fu W, et al. The effect of infraorbital nerve block on emergence agitation in children undergoing cleft lip surgery under general anesthesia with sevoflurane. Paediatr Anaesth. 2015;25 (9):906-910.

22. Yu D, Chai W, Sun X, et al. Emergence agitation in adults: risk factors in 2000 patients. Can J Anaesth. 2010;57(9):843-848. doi:10.1007/s12630-010-9338-9

23. Gerbershagen HJ, Aduckathil S, van Wijck AJM, et al. Pain intensity on the first day after surgery: a prospective cohort study comparing 179 surgical procedures. Anesthesiology. 2013;118(4):934-944. doi:10.1097/ALN.0b013e31828866b3

24. Kim JH. Mechanism of emergence agitation induced by sevoflurane anesthesia. Korean J Anesthesiol. 2011;60(2):73-74. doi:10.4097/ kjae.2011.60.2.73

25. Ibrahim M, Elnabtity AM, Keera A. Efficacy of external nasal nerve block following nasal surgery: a randomised, controlled trial. Anaesthesist. 2018;67(3):188-197. doi:10.1007/s00101-018-0410-0

26. Kanakaraj M, Shanmugasundaram N, Chandramohan M, et al. Regional anesthesia in faciomaxillary and oral surgery. $J$ Pharm Bioallied Sci. 2012;4(Suppl 2):S264-S269. doi:10.4103/09757406.100247

27. Moskovitz JB, Sabatino F. Regional nerve blocks of the face. Emerg Med Clin North Am. 2013;31(2):517-527. doi:10.1016/j. emc.2013.01.003

management system is completely online and includes a very quick and fair peer-review system, which is all easy to use. Visit http:// www.dovepress.com/testimonials.php to read real quotes from published authors. 